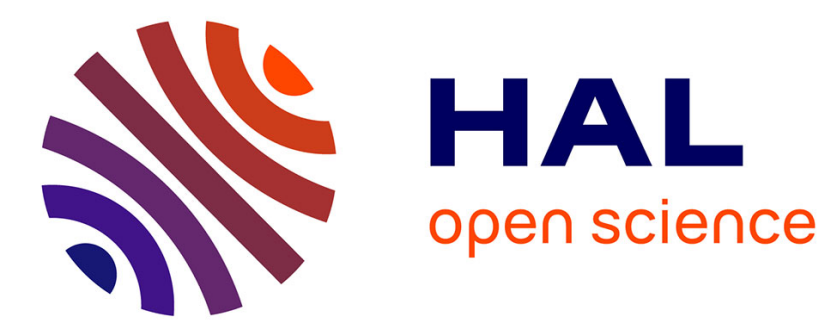

\title{
HOMOGENEITY FOR TIME-DELAY SYSTEMS
}

Denis Efimov, Wilfrid Perruquetti

\section{To cite this version:}

Denis Efimov, Wilfrid Perruquetti. HOMOGENEITY FOR TIME-DELAY SYSTEMS. IFAC WC 2011, Aug 2011, Milan, Italy. pp.1-6. hal-00628890

\section{HAL Id: hal-00628890 https://hal.science/hal-00628890}

Submitted on 4 Oct 2011

HAL is a multi-disciplinary open access archive for the deposit and dissemination of scientific research documents, whether they are published or not. The documents may come from teaching and research institutions in France or abroad, or from public or private research centers.
L'archive ouverte pluridisciplinaire HAL, est destinée au dépôt et à la diffusion de documents scientifiques de niveau recherche, publiés ou non, émanant des établissements d'enseignement et de recherche français ou étrangers, des laboratoires publics ou privés. 


\title{
HOMOGENEITY FOR TIME-DELAY SYSTEMS
}

\author{
Efimov D. ${ }^{1}$, Perruquetti W. ${ }^{2,3}$ \\ ${ }^{1}$ University of Bordeaux, IMS-lab, Automatic control group \\ 351 cours de la libération, 33405 Talence, France \\ denis.efimov@ims-bordeaux.fr \\ ${ }^{2}$ LAGIS (UMR-CNRS 8146), Ecole Centrale de Lille, BP 48, Cité Scientifique, 59651 \\ Villeneuve-d'Ascq, France \\ ${ }^{3}$ ALIEN project at INRIA - LNE, Parc Scientifique de la Haute Borne 40, \\ avenue Halley Bât.A, Park Plaza 59650 Villeneuve d'Ascq, France \\ wilfrid.perruquetti@,inria.fr
}

\begin{abstract}
The notion of homogeneity is extended to the time-delay nonlinear systems. It is shown that under some conditions the stability of homogeneous functional systems on a sphere implies the global stability of the system. The notion of local homogeneity is introduced, the relations between stability of the locally approximating dynamics and the original time-delay system are established. An example of the proposed theory application is given.
\end{abstract}

\section{INTRODUCTION}

For nonlinear systems, behavior of a homogeneous system trajectories can be extended to the whole state space based on their behavior on a suitably defined sphere around the origin (Rothschild and Stein, 1976). Thus the state vector rescaling does not change the system behavior. As it has been shown during the last two decades this property can be used for stability analysis (Andrieu, et al., 2008; Bacciotti and Rosier, 2001; Hermes, 1991b; Hong, 2002; Rosier, 1992), systems approximation (Hermes, 1991a), stabilization (Bhat and Bernstein, 2001; Grüne, 2000; Kawski, 1991; Moulay and Perruquetti, 2006; Sepulchre and Aeyels, 1996) and observation (Andrieu, et al., 2008). Analysis and synthesis of homogeneous systems is a little bit simpler, for example, the Lyapunov function has to be constructed on the unit sphere only (on the whole state space it can extended using rescaling). Moreover, it has been shown that for stability/instability analysis the Lyapunov function of a homogeneous system is also homogeneous (Rosier, 1992; Efimov and Perruquetti, 2010).

In the work (Andrieu, et al., 2008) the homogeneity in the bilimit has been introduced, that is homogeneity with different weights and approximating functions at a vicinity of the origin and far outside. Recently the bi-limit homogeneity has been extended to the local homogeneity (Efimov and Perruquetti, 2010), when the system is similar to a homogeneous one on a sphere only. In this case the homogeneous systems theory allows us to analyze locally the original nonlinear system behavior.

All this theory has been developed for continuous timeinvariant nonlinear dynamical systems. Another important class of systems includes ones described by differential equations with time-delayed states. The presence of delays is usual in many applications (Chiasson and Loiseau, 2007; Richard, 2003). Analysis of the delay influence on the system stability is critical for many natural and human-developed systems (Gu, et al., 2003; Kolmanovsky and Nosov, 1986). The goal of this work is to extend homogeneity approach to the nonlinear time-delay systems. The problem is that the former ones operate in the infinite dimensional state space. Extension of the standard results (Rosier, 1992) to this class of systems needs complete revision of the homogeneous systems apparatus. In (Asl and Ulsoy, 2000; Dublik, 1997) the homogeneous (linear) delay differential equation have been studied, in (Bokharaie, et al., 2010) the cooperative and homogeneous systems have been analyzed. In all these works the homogeneity theory has not been extended to the functional spaces, the papers are based on homogeneity of nondelayed parts of differential equations.

The outline of this work is as follows. The preliminary definitions and the system equations are given in Section 2. The homogeneous norm, an extended definition of homogeneity for time-delay systems and sufficient stability conditions are presented in Section 3. The local homogeneity theory is studied in Section 4. An example is presented in Section 5.

\section{PRELIMINARIES}

Consider the autonomous functional differential equation:

$$
d \mathbf{x}(t) / d t=\mathbf{f}\left[\mathbf{x}_{\tau}(t)\right], t \geq 0,
$$

where $\quad \mathbf{x} \in R^{n} \quad$ is the state vector, $\quad \mathbf{x}_{\tau}(t)=\mathbf{x}(t+s)$, $-\tau \leq s \leq 0 \in C^{n}[-\tau, 0]$ is the extended (lifted) state vector (we denote by $C^{n}[a, b], 0 \leq a<b \leq+\infty$ the Banach space of continuous functions $\varphi:[a, b] \rightarrow R^{n}$ with the norm $\|\varphi\|=\sup _{a \leq \varsigma \leq b}|\varphi(\varsigma)|$, where $|\cdot|$ is the standard Euclidean norm); $\mathbf{f}: C^{n}[-\tau, 0] \rightarrow R^{n}$ is a locally Lipschitz continuous function, $\mathbf{f}(0)=0$. We assume that all solutions of the sys- 
tem (1) satisfy the initial condition $\mathbf{x}_{\tau}(0)=\mathbf{x}_{0} \in C^{n}[-\tau, 0]$. It is known from the theory of functional differential equations (Gu, et al., 2003), that under above assumptions the system (1) has a unique solution $\mathbf{x}\left(t, \mathbf{x}_{0}\right)$ satisfying initial condition $\mathbf{x}_{0}$, which is defined on some finite interval $[0, T)$. The upper right hand Dini derivative of the Lipschitz continuous functional $V: C^{n}[-\tau, 0] \rightarrow R$ along the system (1) solutions is defined as follows for any $\varphi \in C^{n}[-\tau, 0]$ :

$$
D^{+} V(\varphi)=\limsup _{h \rightarrow 0^{+}} \frac{1}{h}\left[V\left(\varphi_{h}\right)-V(\varphi)\right],
$$

where $\varphi_{h} \in C^{n}[-\tau, 0]$ for $0<h<\tau$ is given by

$$
\varphi_{h}= \begin{cases}\varphi(\theta+h), & \theta \in[-\tau,-h) \\ \varphi(0)+\mathbf{f}(\varphi)(\theta+h), & \theta \in[-h, 0] .\end{cases}
$$

The notation $D V\left[\mathbf{x}_{\tau}(0)\right] \mathbf{f}\left(\mathbf{x}_{\tau}\right)$ stands for directional derivative of the continuously differentiable function $V: R^{n} \rightarrow R$ with respect to the vector field $\mathbf{f}$.

Qualify a continuous function $\sigma: R_{+} \rightarrow R_{+}$belongs to class $\mathcal{K}$ if it is strictly increasing and $\sigma(0)=0$; it belongs to class $K_{\infty}$ if it is also radially unbounded.

\section{HOMOGENEITY}

For any $r_{i}>0, i=\overline{1, n}$ and $\lambda \geq 0$ define the dilation matrix $\boldsymbol{\Lambda}_{\mathbf{r}}=\operatorname{diag}\left\{\lambda^{r_{i}}\right\}_{i=1}^{n}$ and the vector of weights $\mathbf{r}=\left[r_{1} \ldots r_{n}\right]^{T}$.

For any $r_{i}>0, i=\overline{1, n}$ and $\mathbf{x} \in R^{n}$ the homogeneous norm can be defined as follows

$$
|\mathbf{x}|_{\mathbf{r}}=\left(\sum_{i=1}^{n}\left|x_{i}\right|^{\rho / r_{i}}\right)^{1 / \rho}, \rho=\prod_{i=1}^{n} r_{i} .
$$

For all $\mathbf{x} \in R^{n}$, its Euclidean norm $|\mathbf{x}|$ is related with the homogeneous one via some constants $\underline{\sigma}_{\mathbf{r}}, \bar{\sigma}_{\mathbf{r}} \in R_{+}$:

$$
\underline{\sigma}_{\mathbf{r}}|\mathbf{x}|_{\mathbf{r}} \leq|\mathbf{x}| \leq \bar{\sigma}_{\mathbf{r}}|\mathbf{x}|_{\mathbf{r}},
$$

where $\underline{\sigma}_{\mathbf{r}}, \bar{\sigma}_{\mathbf{r}}$ define the Euclidean norm deviations with respect to the homogeneous norm. The homogeneous norm has an important property that $\left|\Lambda_{\mathbf{r}} \mathbf{x}\right|_{\mathbf{r}}=\lambda|\mathbf{x}|_{\mathbf{r}}$ for all $\mathbf{x} \in R^{n}$. Define $S_{\mathbf{r}}=\left\{\mathbf{x} \in R^{n}:|\mathbf{x}|_{\mathbf{r}}=1\right\}$.

Since the dilation matrix $\Lambda_{\mathbf{r}}$ transformation is linear, it can be applied to functional arguments. Indeed, for any $r_{i}>0$, $i=\overline{1, n}$ and $\varphi \in C^{n}[a, b], 0 \leq a<b \leq+\infty$ the homogeneous norm can be defined as follows

$$
\|\varphi\|_{\mathbf{r}}=\left(\sum_{i=1}^{n}\left\|\varphi_{i}\right\|^{\rho / r_{i}}\right)^{1 / \rho}, \rho=\prod_{i=1}^{n} r_{i} .
$$

L e m m a 1 . For all $\varphi \in C^{n}[a, b]$, there exist two functions $\underline{\rho}_{\mathbf{r}}, \bar{\rho}_{\mathbf{r}} \in K_{\infty}$ such that

$$
\underline{\rho}_{\mathbf{r}}\left(\|\varphi\|_{\mathbf{r}}\right) \leq\|\varphi\| \leq \bar{\rho}_{\mathbf{r}}\left(\|\varphi\|_{\mathbf{r}}\right) .
$$

Pro of. By definition

$$
\|\varphi\|=\sup _{a \leq \varsigma \leq b}|\varphi(\varsigma)|=\sup _{a \leq \varsigma \leq b} \sqrt{\sum_{i=1}^{n}\left|\varphi_{i}(\varsigma)\right|^{2}} .
$$

Thus $\|\varphi\| \leq \sqrt{\sum_{i=1}^{n}\left\|\varphi_{i}\right\|^{2}}$ and $\bar{\rho}(s)=\bar{c}_{\rho} s^{0.5 \rho \max _{1 \leq i \leq n} r_{i}}$ for some $\bar{c}_{\rho}>0$ dependent on $\rho, \sqrt{\sum_{i=1}^{n}\left\|\varphi_{i}\right\|^{2}} \leq n\|\varphi\|$ and $\underline{\rho}(s)=\underline{c}_{\rho} n^{-1} s^{0.5 \rho \min _{1 \leq i \leq n} r_{i}}, \underline{c}_{\rho}>0$.

Therefore, the proposed homogeneous norm is equivalent to the supremum norm in $C^{n}[a, b]$. The homogeneous norm in the Banach space has the same important property that $\left\|\boldsymbol{\Lambda}_{\mathbf{r}} \varphi\right\|_{\mathbf{r}}=\lambda\|\varphi\|_{\mathbf{r}}$ for all $\varphi \in C^{n}[a, b]$. Define the corresponding unit sphere $S_{\mathbf{r}}=\left\{\varphi \in C^{n}[-\tau, 0]:\|\varphi\|_{\mathbf{r}}=1\right\}$.

Definition 1 . The function $g: C^{n}[-\tau, 0] \rightarrow R$ is called $\mathbf{r}$-homogeneous $\left(r_{i}>0, i=\overline{1, n}\right)$, if for any $\varphi \in C^{n}[-\tau, 0]$ the relation

$$
g\left(\boldsymbol{\Lambda}_{\mathbf{r}} \varphi\right)=\lambda^{d} g(\varphi)
$$

holds for some $d \geq 0$ and all $\lambda \geq 0$.

The function $\mathbf{f}: C^{n}[-\tau, 0] \rightarrow R^{n}$ is called $\mathbf{r}$-homogeneous $\left(r_{i}>0, i=\overline{1, n}\right)$, if for any $\varphi \in C^{n}[-\tau, 0]$ the relation

$$
\mathbf{f}\left(\boldsymbol{\Lambda}_{\mathbf{r}} \varphi\right)=\lambda^{d} \boldsymbol{\Lambda}_{\mathbf{r}} \mathbf{f}(\varphi)
$$

holds for some $d \geq-\min _{1 \leq i \leq n} r_{i}$ and all $\lambda \geq 0$.

The constant $d$ is called the degree of homogeneity. The introduced notion of homogeneity in $C^{n}[-\tau, 0]$ is reduced to the standard one in $R^{n}$ (Rosier, 1992) under vector argument substitution. The advantage of homogeneous systems described by non linear ordinary differential equations is that analysis of their stability can be performed on the unit sphere $S_{\mathbf{r}}$ only (Rosier, 1992) (the homogeneous system trajectories have similar behavior on any sphere defined by the norm $\left.|\cdot|_{\mathbf{r}}\right)$. It has been also shown that asymptotically stable/unstable homogeneous systems always have homogeneous Lyapunov functions (Efimov and Perruquetti, 2010; Rosier, 1992). In this work we would like to prove a similar (sufficient only) result for time-delay homogeneous systems.

For time-delay systems there exist two main techniques for stability analysis based on the Lyapunov approach. The first one is based on the Lyapunov-Krasovskii functional, another one is the Razumikhin stability theorem $(\mathrm{Gu}$, et al., 2003; Kolmanovsky and Nosov, 1986). Unfortunately, due to peculiarities of the directional derivatives for functionals (see the definition above) the Lyapunov-Krasovskii approach is hard to develop using homogeneity. To explain the problem consider the following example. Let the functions $\mathbf{f}_{0}: R^{n} \rightarrow R^{n}$, $\mathbf{f}_{1}: R^{n} \rightarrow R^{n}, \quad g_{0}: R^{n} \rightarrow R_{+}, \quad g_{1}: R^{n} \rightarrow R_{+} \quad$ be $\quad \mathbf{r}-$ homogeneous (with the same $r_{i}>0, i=\overline{1, n}$ and similar de- 
gree $d \geq-\min _{1 \leq i \leq n} r_{i}$ for $\mathbf{f}_{0}, \mathbf{f}_{1}$ and $v \geq 0$ for $\left.g_{0}, g_{1}\right)$. For $\mathbf{f}\left[\mathbf{x}_{\tau}(t)\right]=\mathbf{f}_{0}[\mathbf{x}(t)]+\mathbf{f}_{1}[\mathbf{x}(t-\tau)]$ in (1) consider the LyapunovKrasovskii functional

$$
V\left[\mathbf{x}_{\tau}(t)\right]=g_{0}[\mathbf{x}(t)]+\int_{t-\tau}^{t} g_{1}[\mathbf{x}(s)] d s,
$$

that satisfies the required homogeneity conditions. Assume that the structural condition

$$
\partial g_{0}(\mathbf{x}) /\left.\partial \mathbf{x}\right|_{\mathbf{x}=\mathbf{x}(t)} \mathbf{f}_{1}[\mathbf{x}(t-\tau)] \leq g_{1}[\mathbf{x}(t-\tau)]+g_{2}\left[\mathbf{x}_{2}(t)\right]
$$

holds for some function $g_{2}: R^{n} \rightarrow R$, then verification of the derivative $\dot{V}$ negative definiteness can be performed for $\mathbf{x}(t) \in S_{\mathbf{r}}$ (that is relatively simple):

$$
\begin{aligned}
\dot{V}= & \partial g_{0}(\mathbf{x}) /\left.\partial \mathbf{x}\right|_{\mathbf{x}=\mathbf{x}(t)}\left\{\mathbf{f}_{0}[\mathbf{x}(t)]+\mathbf{f}_{1}[\mathbf{x}(t-\tau)]\right\}+ \\
& g_{1}[\mathbf{x}(t)]-g_{1}[\mathbf{x}(t-\tau)] \leq \\
\leq & \partial g_{0}(\mathbf{x}) /\left.\partial \mathbf{x}\right|_{\mathbf{x}=\mathbf{x}(t)} \mathbf{f}_{0}[\mathbf{x}(t)]+g_{1}[\mathbf{x}(t)]+g_{2}[\mathbf{x}(t)] .
\end{aligned}
$$

Let for $\mathbf{x}(t) \in S_{\mathbf{r}}$ the above expression verifies $\dot{V}<0$. Consider the dilation transformation $\mathbf{z}=\Lambda_{\mathbf{r}} \mathbf{x}$ influence on $\dot{V}$ :

$$
\begin{gathered}
\dot{V}(\mathbf{z})=\dot{V}\left(\boldsymbol{\Lambda}_{\mathbf{r}} \mathbf{x}\right)=\lambda^{d+v} \partial g_{0}(\mathbf{x}) /\left.\partial \mathbf{x}\right|_{\mathbf{x}=\mathbf{x}(t)}\left\{\mathbf{f}_{0}[\mathbf{x}(t)]+\right. \\
\left.\mathbf{f}_{1}[\mathbf{x}(t-\tau)]\right\}+\lambda^{v}\left\{g_{1}[\mathbf{x}(t)]-g_{1}[\mathbf{x}(t-\tau)]\right\} .
\end{gathered}
$$

Thus this basic for homogeneous systems transformation scales differently the parts proportional to $g_{0}$ and $g_{1}$, therefore in general case for some $\lambda \geq 0$ it could be $\dot{V}>0$.

Since the Razumikhin approach, based on LyapunovRazumikhin functions (Gu, et al., 2003) defined on $R^{n}$, gives a point-wise sufficient criteria for stability (not a functional one), it allows us to develop this approach using homogeneous arguments. Note, that if a function $V: R^{n} \rightarrow R_{+}$ is positive definite and radially unbounded, then there are functions $\alpha_{1}, \alpha_{2} \in K_{\infty}$ such that $\alpha_{1}(|\mathbf{x}|) \leq V(\mathbf{x}) \leq \alpha_{2}(|\mathbf{x}|)$ for all $\mathbf{x} \in R^{n}$.

$\mathrm{Th}$ e o r e m 1. Let the function $\mathbf{f}$ in (1) be $\mathbf{r}$-homogeneous with degree $d$ and there exist $\mathbf{r}$-homogeneous continuously differentiable Lyapunov-Razumikhin function $V: R^{n} \rightarrow R_{+}$ with degree $v, v+d>0$ such that

(i) there exist functions $\alpha, \gamma \in \mathcal{K}\left(\gamma(s)>s\right.$ for all $\left.s \in R_{+}\right)$ such that for all $\phi \in S_{\mathbf{r}}$

$\max _{\theta \in[-\tau, 0]} V[\phi(\theta)]<\gamma\{V[\phi(0)]\} \Rightarrow D V[\phi(0)] \mathbf{f}(\phi) \leq-\alpha(|\phi(0)|) ;$

(ii) there exists function $\gamma^{\prime} \in \mathcal{K}$ such that $\lambda \gamma(s) \leq \gamma^{\prime}(\lambda s)$ for all $s, \lambda \in R_{+}$.

Then the system (1) is globally asymptotically stable.

Proof. The dilation transformation $\varphi=\Lambda_{\mathbf{r}} \phi$ connects any $\varphi \in C^{n}[-\tau, 0]$ with some $\phi \in S_{\mathbf{r}}$ for properly chosen $\lambda \geq 0$. Let us multiply the left hand part of the condition (i) by $\lambda^{v}$ and the right hand part by $\lambda^{v+d}$ :

$$
\begin{gathered}
\max _{\theta \in[-\tau, 0]} \lambda^{v} V[\phi(\theta)]<\lambda^{v} \gamma\{V[\phi(0)]\} \Rightarrow \\
\lambda^{v+d} D V[\phi(0)] \mathbf{f}(\phi) \leq-\lambda^{v+d} \alpha(|\phi(0)|),
\end{gathered}
$$

the inequalities and relations should not be changed for $\lambda \geq 0$. Owing the function $\gamma$ properties, the inequality $\lambda^{v} \gamma\{V[\phi(0)]\} \leq \gamma^{\prime}\left\{\lambda^{v} V[\phi(0)]\right\} \quad$ holds. Formally $\inf _{\phi \in S_{\mathbf{r}}}\{\alpha(|\phi(0)|)\}=0$, however having in mind that $\phi$ is a continuous function and a solution of (1), there exists

$$
a=-\inf _{\phi \in S_{\mathrm{r}}, \max _{\theta \in[-\tau, 0]} V[\phi(\theta)]<\gamma\{V[\phi(0)]\}}\{\alpha(|\phi(0)|)\}
$$

and $a>0$. Therefore, due to homogeneity of the functions $\mathbf{f}$ and $V$ we have:

$$
\begin{gathered}
\max _{\theta \in[-\tau, 0]} V\left[\boldsymbol{\Lambda}_{\mathbf{r}} \phi(\theta)\right]<\gamma^{\prime}\left\{V\left[\boldsymbol{\Lambda}_{\mathbf{r}} \phi(0)\right]\right\} \Rightarrow \\
D V\left[\boldsymbol{\Lambda}_{\mathbf{r}} \phi(0)\right] \mathbf{f}\left(\boldsymbol{\Lambda}_{\mathbf{r}} \phi\right) \leq-\lambda^{v+d} a,
\end{gathered}
$$

or equivalently $\left(\lambda=\|\varphi\|_{\mathbf{r}} \geq|\varphi(0)|_{\mathbf{r}} \geq \bar{\sigma}_{\mathbf{r}}^{-1}|\varphi(0)|\right)$,

$$
\begin{gathered}
\max _{\theta \in[-\tau, 0]} V[\varphi(\theta)]<\gamma^{\prime}\{V[\varphi(0)]\} \Rightarrow \\
D V[\varphi(0)] \mathbf{f}(\varphi) \leq-\alpha^{\prime}(|\varphi(0)|),
\end{gathered}
$$

where the function $\alpha^{\prime}(s)=\left(\bar{\sigma}_{\mathbf{r}}^{-1} s\right)^{v+d} a$ is from class $\mathcal{K}$ due to $v+d>0$. Therefore, if Razumikhin arguments are true for $\phi \in S_{\mathbf{r}}$, then they hold for any $\varphi \in C^{n}[-\tau, 0]$, that implies the global asymptotic stability of (1).

The condition (i) imposed in Theorem 1 on the system (1) behavior is the conventional Razumikhin condition (except in the homogeneous case it can be verified on the sphere $S_{\mathbf{r}}$ only). The constraint (ii) on existence of the function $\gamma^{\prime}$ is new. Roughly speaking this requirement says that the function $\gamma$ has to be "homogeneous" with degree 1. Another explanation is that the function $\gamma$ is globally Lipschitz. For instance, this is the case if there exists $1<k_{1}<k_{2}<+\infty$ such that $k_{1} s \leq \gamma(s) \leq k_{2} s$.

Remark 1 . Note that the full derivative $D V[\phi(0)] \mathbf{f}(\phi)$ is a function of $\phi \in S_{\mathbf{r}}$, however under the condition $\max _{\theta \in[-\tau, 0]} V\left[\Lambda_{\mathbf{r}} \phi(\theta)\right]<\gamma^{\prime}\left\{V\left[\Lambda_{\mathbf{r}} \phi(0)\right]\right\} \quad$ the inequality $D V[\phi(0)] \mathbf{f}(\phi)<m(\phi(0))$ holds for some $m: R^{n} \rightarrow R$. If the function $m$ is $\mathbf{r}$-homogeneous (the functions $V$ and $\mathbf{f}$ possess this property), then the property $m(\phi(0)) \leq-\alpha(|\phi(0)|)$ in (ii) has to be verified for $\phi(0) \in S_{\mathbf{r}}$ only.

The result of Theorem 1 means that the system stability can be checked on the sphere $S_{\mathbf{r}}$ only for homogeneous systems with homogeneous Lyapunov-Razumikhin function. These facts may simplify the function $V$ search and the system analysis applying numerical routines. The drawback is that in the space $C^{n}[-\tau, 0]$ this sphere $S_{\mathbf{r}}$ is a rather complex object, especially the subset of $\phi \in S_{\mathbf{r}}$ where

$$
\max _{\theta \in[-\tau, 0]} V[\varphi(\theta)]<\gamma^{\prime}\{V[\varphi(0)]\} .
$$




\section{LOCAL HOMOGENEITY}

A disadvantage of the global homogeneity introduced so far is that such systems possess the same behavior globally for all $\varphi \in C^{n}[-\tau, 0]$. Thus the homogeneous systems are not really "nonlinear", they have similar diversity of operating modes as linear systems. In fact, the homogeneous systems are a generalization of linear ones from analysis and design points of view. Comparing with other nonlinear systems it is easier to find Lyapunov function for homogeneous ones. Additionally, since the homogeneous systems have global stability property, the local stability analysis at the origin using linearization verifies the global stability in this case. This is why finding a possibility to apply this approach for a broader class of nonlinear systems is very important. An approach to resolve this issue consists in local version of homogeneity introduction as in (Efimov and Perruquetti, 2010).

Definition 2. The function $g: C^{n}[-\tau, 0] \rightarrow R, g(0)=0$ is called $\quad\left(\mathbf{r}, \lambda_{0}, g_{0}\right)$-homogeneous $\quad\left(r_{i}>0, \quad i=\overline{1, n}\right.$; $\left.g_{0}: C^{n}[-\tau, 0] \rightarrow R, g_{0}(0)=0\right)$ iffor any $\varphi \in S_{\mathbf{r}}$ the relation

$$
\lim _{\lambda \rightarrow \lambda_{0}} \lambda^{-d_{0}} g\left(\Lambda_{\mathbf{r}} \varphi\right)-g_{0}(\varphi)=0
$$

holds for some $d_{0} \geq 0$.

The system (1) is called $\left(\mathbf{r}, \lambda_{0}, \mathbf{f}_{0}\right)$-homogeneous $\left(r_{i}>0\right.$, $\left.i=\overline{1, n} ; \mathbf{f}_{0}: C^{n}[-\tau, 0] \rightarrow R^{n}, \mathbf{f}_{0}(0)=0\right)$ if for any $\varphi \in S_{\mathbf{r}}$ the relation

$$
\lim _{\lambda \rightarrow \lambda_{0}} \lambda^{-d_{0}} \boldsymbol{\Lambda}_{\mathbf{r}}^{-1} \mathbf{f}\left(\boldsymbol{\Lambda}_{\mathbf{r}} \varphi\right)-\mathbf{f}_{0}(\varphi)=0
$$

holds for some $d_{0} \geq-\min _{1 \leq i \leq n} r_{i}$.

This property is called local homogeneity (Efimov and Perruquetti, 2010), it allows us to analyze local stability of the system (1) on the basis of the simplified system

$$
d \mathbf{y}(t) / d t=\mathbf{f}_{0}\left[\mathbf{y}_{\tau}(t)\right], t \geq 0,
$$

called the local approximating dynamics for (1). For any $0<\lambda_{0}<+\infty$ the following formulas give a variant of homogeneous approximations $g_{0}$ and $\mathbf{f}_{0}$ choice:

$$
\begin{gathered}
g_{0}(\varphi)=\|\varphi\|_{\mathbf{r}}^{d} \lambda_{0}^{-d_{0}} g\left(\boldsymbol{\Lambda}_{\mathbf{r}, 0} \boldsymbol{\Lambda}_{\|\varphi\|}^{-1} \varphi\right), \\
\mathbf{f}_{0}(\varphi)=\|\varphi\|_{\mathbf{r}}^{d} \lambda_{0}^{-d_{0}} \boldsymbol{\Lambda}_{\|\varphi\|} \boldsymbol{\Lambda}_{\mathbf{r}, 0}^{-1} \mathbf{f}\left(\boldsymbol{\Lambda}_{\mathbf{r}, 0} \boldsymbol{\Lambda}_{\|\varphi\|}^{-1} \varphi\right),
\end{gathered}
$$

where $\boldsymbol{\Lambda}_{\mathbf{r}, 0}=\operatorname{diag}\left\{\lambda_{0}^{r_{i}}\right\}_{i=1}^{n}, \quad \boldsymbol{\Lambda}_{\|\varphi\|}=\operatorname{diag}\left\{\left\|\varphi_{i}\right\|_{0}^{r_{i}}\right\}_{i=1}^{n} \quad$ and $d \geq 0$.

Theorem 2. Let the system (1) be $\left(\mathbf{r}, \lambda_{0}, \mathbf{f}_{0}\right)$ homogeneous for some $r_{i}>0, i=\overline{1, n}$, the function $\mathbf{f}_{0}$ be continuous and $\mathbf{r}$-homogeneous with the degree $d_{0}$ and there exist a $\mathbf{r}$-homogeneous Lyapunov-Razumikhin function $V_{0}: R^{n} \rightarrow R_{+}$with the degree $v_{0}, v_{0}+d_{0}>0$ such that:

(i) there exist functions $\alpha, \gamma \in \mathcal{K}\left(\gamma(s)>s\right.$ for all $\left.s \in R_{+}\right)$ such that for all $\phi \in S_{\mathbf{r}}$

$$
\begin{gathered}
\max _{\theta \in[-\tau, 0]} V_{0}[\phi(\theta)]<\gamma\left\{V_{0}[\phi(0)]\right\} \Rightarrow \\
D V_{0}[\phi(0)] \mathbf{f}_{0}(\phi) \leq-\alpha(|\phi(0)|) ;
\end{gathered}
$$

(ii) there exists function $\gamma^{\prime} \in \mathcal{K}$ such that $\lambda \gamma(s) \leq \gamma^{\prime}(\lambda s)$ for all $s, \lambda \in R_{+}$.

\section{Then}

1) if $\lambda_{0}=0$, then there exists $0<\bar{\lambda}_{\varepsilon}$ such that the system (1) is locally asymptotically stable with the domain of asymptotic stability containing the set

$$
X_{0}=\left\{\varphi \in C^{n}[-\tau, 0]:\|\varphi\| \leq \alpha_{1}^{-1} \circ \alpha_{2} \circ \underline{\rho}_{\mathbf{r}}\left(\bar{\lambda}_{\varepsilon}\right)\right\} ;
$$

2) if $\lambda_{0}=+\infty$, then there exists $0<\underline{\lambda} \varepsilon<+\infty$ such that the system (1) is globally asymptotically stable with respect to forward invariant set

$$
X_{\infty}=\left\{\varphi \in C^{n}[-\tau, 0]:\|\varphi\| \leq \alpha_{1}^{-1} \circ \alpha_{2} \circ \bar{\rho}_{\mathbf{r}}\left(\underline{\lambda}_{\varepsilon}\right)\right\} ;
$$

3) if $0<\lambda_{0}<+\infty$, then there exist $0<\underline{\lambda}_{\varepsilon} \leq \lambda_{0} \leq \bar{\lambda}_{\varepsilon}<+\infty$ such that the system (1) is asymptotically stable with respect to the forward invariant set $X_{\infty}$ with region of attraction

$$
\begin{gathered}
X=\left\{\varphi \in C^{n}[-\tau, 0]: \alpha_{1}^{-1} \circ \alpha_{2} \circ \bar{\rho}_{\mathbf{r}}\left(\underline{\lambda}_{\varepsilon}\right)<\|\varphi\|\right. \\
\left.<\alpha_{1}^{-1} \circ \alpha_{2} \circ \underline{\rho}_{\mathbf{r}}\left(\bar{\lambda}_{\varepsilon}\right)\right\}
\end{gathered}
$$

provided that the set $X$ is connected and $X \neq \varnothing$.

Proof. For the system (1) the coordinates transformation $\varphi=\Lambda_{\mathbf{r}} \phi$ connects any $\varphi \in C^{n}[-\tau, 0]$ with some $\phi \in S_{\mathbf{r}}$ for suitably chosen $\lambda \geq 0$. According to definition of the function $V_{0}$ we have:

$$
\begin{aligned}
& D V_{0}[\varphi(0)] \mathbf{f}(\varphi)=D V_{0}\left[\boldsymbol{\Lambda}_{\mathbf{r}} \phi(0)\right] \mathbf{f}\left(\boldsymbol{\Lambda}_{\mathbf{r}} \phi\right)= \\
& =D V_{0}\left[\boldsymbol{\Lambda}_{\mathbf{r}} \phi(0)\right]\left\{\mathbf{f}_{0}\left(\boldsymbol{\Lambda}_{\mathbf{r}} \phi\right)+\left[\mathbf{f}\left(\boldsymbol{\Lambda}_{\mathbf{r}} \phi\right)-\mathbf{f}_{0}\left(\boldsymbol{\Lambda}_{\mathbf{r}} \phi\right)\right]\right\}= \\
& =\lambda^{d_{0}+v_{0}} D V_{0}[\phi(0)] \mathbf{f}_{0}(\phi)+\lambda^{v_{0}} D V_{0}[\phi(0)] \boldsymbol{\Lambda}_{\mathbf{r}}^{-1}\left\{\mathbf{f}\left(\boldsymbol{\Lambda}_{\mathbf{r}} \phi\right)-\right. \\
& \left.\lambda^{d_{0}} \boldsymbol{\Lambda}_{\mathbf{r}} \mathbf{f}_{0}(\phi)\right\}=\lambda^{d_{0}+v_{0}} D V_{0}[\phi(0)]\left\{\mathbf{f}_{0}(\phi)+\right. \\
& \left.\left[\lambda^{-d_{0}} \boldsymbol{\Lambda}_{\mathbf{r}}^{-1} \mathbf{f}\left(\boldsymbol{\Lambda}_{\mathbf{r}} \phi\right)-\mathbf{f}_{0}(\phi)\right]\right\} .
\end{aligned}
$$

Due to continuity of the functions $\mathbf{f}, \mathbf{f}_{0}$ and the local homogeneity property definition for any $\varepsilon>0$ there exist $\underline{\lambda}_{\varepsilon} \leq \lambda_{0} \leq \bar{\lambda}_{\varepsilon}$ such that

$$
\sup _{\phi \in S_{\mathbf{r}}}\left|D V_{0}[\phi(0)]\left\{\lambda^{-d_{0}} \boldsymbol{\Lambda}_{\mathbf{r}}^{-1} \mathbf{f}\left(\boldsymbol{\Lambda}_{\mathbf{r}} \phi\right)-\mathbf{f}_{0}(\phi)\right\}\right| \leq \varepsilon
$$

for all $\lambda \in\left(\underline{\lambda}_{\varepsilon}, \bar{\lambda}_{\varepsilon}\right)$. From (i) we know that under the condition $\max _{\theta \in[-\tau, 0]} V_{0}[\phi(\theta)]<\gamma\left\{V_{0}[\phi(0)]\right\} \quad$ inequality $D V_{0}[\phi(0)] \mathbf{f}_{0}(\phi) \leq-\alpha(|\phi(0)|) \quad$ is satisfied. Since $\inf _{\phi \in S_{\mathrm{r}}}\{\alpha(|\phi(0)|)\}=0$ it seems that the additive disturbance caused by the dilation transformation can not be attenuated by $\alpha$. However, the condition

$$
V_{0}[\phi(0)] \leq \max _{\theta \in[-\tau, 0]} V_{0}[\phi(\theta)]<\gamma\left\{V_{0}[\phi(0)]\right\}
$$

excludes from consideration some functions, in particular those with $\phi(0)=0$. Moreover, taking in mind that $\phi$ is a 
continuous function and a solution of (1), there is

$$
a=-\inf _{\phi \in S_{\mathrm{r}}, \max _{\theta \in[-\tau, 0]} V_{0}[\phi(\theta)]<\gamma\left\{V_{0}[\phi(0)]\right\}}\{\alpha(|\phi(0)|)\}
$$

and $a>0$. Let the constants $\underline{\lambda}_{\varepsilon}, \bar{\lambda}_{\varepsilon}$ be chosen to ensure that $a>\varepsilon$. Multiplying the inequality $V_{0}[\phi(\theta)]<\gamma\left\{V_{0}[\phi(0)]\right\}$ on $\lambda^{v_{0}}$ we get that it is equivalent to $V_{0}[\varphi(\theta)]<\gamma^{\prime}\left\{V_{0}[\varphi(0)]\right\}$ for all $\theta \in[-\tau, 0]$. Then from (i), (ii) the following property holds for $\alpha^{\prime}(s)=\left(\bar{\sigma}_{\mathbf{r}}^{-1} s\right)^{v_{0}+d_{0}}(a-\varepsilon)$ :

$$
\begin{gathered}
\max _{\theta \in[-\tau, 0]} V_{0}[\varphi(\theta)]<\gamma^{\prime}\left\{V_{0}[\varphi(0)]\right\} \Rightarrow \\
D V_{0}[\varphi(0)] \mathbf{f}_{0}(\varphi) \leq-\alpha^{\prime}(|\varphi(0)|),
\end{gathered}
$$

where $\varphi=\Lambda_{\mathbf{r}} \phi, \phi \in S_{\mathbf{r}}$ and $\lambda \in\left(\underline{\lambda}_{\varepsilon}, \bar{\lambda}_{\varepsilon}\right)$, i.e. for all $\mathbf{x}_{\tau} \in X_{\mathbf{r}}=\left\{\mathbf{x} \in C^{n}[-\tau, 0]: \underline{\lambda}_{\varepsilon}<\left\|\mathbf{x}_{\tau}\right\|_{\mathbf{r}}<\bar{\lambda}_{\varepsilon}\right\} \quad$ in the system (1). If $\lambda_{0}=0$, then clearly $\underline{\lambda}_{\varepsilon}=0$ and the system is locally asymptotically stable with the domain of asymptotic stability containing the set $X_{0}(\mathrm{Gu}$, et al., 2003; Kolmanovsky and Nosov, 1986). If $\lambda_{0}=+\infty$, then $\bar{\lambda}_{\varepsilon}=+\infty$ and the function $V_{0}$ has strictly negative time derivative for the system (1) into the set $R^{n} \backslash X_{\infty}$. Thus the set $X_{\infty}$ is forward invariant for (1) and borrowing arguments from $(\mathrm{Gu}$, et al., 2003; Lin, et al., 1996), these facts imply the global asymptotic stability of the system (1) with respect to the set $X_{\infty}$. Finally, let $0<\lambda_{0}<+\infty$ and the set $X$ be not empty, then the function $V_{0}$ is strictly decreasing into the set $X$ and all trajectories $\mathbf{x}\left(t, \mathbf{x}_{0}\right)$ with initial conditions $\mathbf{x}_{0} \in X$ reach for the set $X_{\infty}$, that implies the desired conclusion.

This result establishes the links between different variants of local homogeneity with stable approximating dynamics and the stability properties of the original system similarly (Efimov and Perruquetti, 2010). In (Efimov and Perruquetti, 2010) the case of unstable approximating dynamics has been also studied. Note that Remark 1 is valid for the condition (ii) verification.

Corollary 1. Let the system (1) be $\left(\mathbf{r}, \lambda_{0}, \mathbf{f}_{0}\right)$ homogeneous for some $r_{i}>0, i=\overline{1, n}$, the function $\mathbf{f}_{0}: R^{n} \rightarrow R^{n}$ be continuous and $\mathbf{r}$-homogeneous with the degree $d_{0}$ and there exist a $\mathbf{r}$-homogeneous LyapunovRazumikhin function $V_{0}: R^{n} \rightarrow R_{+}$with the degree $v_{0}$, $v_{0}+d_{0}>0$ such that $D V_{0}(\mathbf{x}) \mathbf{f}_{0}(\mathbf{x}) \leq-\alpha(|\mathbf{x}|)$ for all $\mathbf{x} \in S_{\mathbf{r}}$. Then all conclusions of Theorem 2 hold.

Proof. If $\mathbf{f}_{0}: R^{n} \rightarrow R^{n}$, then the conditions (i) and (ii) of Theorem 2 can be reduced to $D V_{0}(\mathbf{x}) \mathbf{f}_{0}(\mathbf{x}) \leq-\alpha(|\mathbf{x}|)$ for all $\mathbf{x} \in S_{\mathbf{r}}$ (the functions $\mathbf{f}_{0}$ and $V_{0}$ are homogeneous).

\section{EXAMPLE}

Consider the system:

$$
\begin{gathered}
\dot{x}_{1}(t)=-x_{1}(t)+x_{2}(t)-x_{1}^{3}(t) ; \\
\dot{x}_{2}(t)=-k x_{2}(t)-x_{1}(t)+a x_{2}(t-\tau)-x_{2}^{3}(t),
\end{gathered}
$$

where $k, a$ are some positive parameters, $\tau>0$ is a fixed time delay. This system is locally homogeneous in the bilimit:

$$
\begin{gathered}
\lambda_{1}=0, \mathbf{r}_{1}=\left[\begin{array}{ll}
0.5 & 0.5
\end{array}\right], \\
\mathbf{f}_{1}\left(\mathbf{x}_{\tau}\right)=\left[\begin{array}{ll}
-x_{1}(t)+x_{2}(t) & -k x_{2}(t)-x_{1}(t)+a x_{2}(t-\tau)
\end{array}\right]^{T}, \\
d_{1}=0, V_{1}(\mathbf{x})=x_{1}^{2}(t)+x_{2}^{2}(t), v_{1}=1 ; \\
\lambda_{2}=+\infty, \mathbf{r}_{2}=\left[\begin{array}{ll}
1 & 1
\end{array}\right], \mathbf{f}_{2}\left(\mathbf{x}_{\tau}\right)=\left[\begin{array}{ll}
-x_{1}^{3}(t) & -x_{2}^{3}(t)
\end{array}\right]^{T}, \\
d_{2}=2, V_{2}(\mathbf{x})=x_{1}^{2}(t)+x_{2}^{2}(t), v_{2}=1 .
\end{gathered}
$$

Straightforward calculations show that

$$
\begin{aligned}
\dot{V}_{1} & =2\left[-x_{1}^{2}(t)-k x_{2}^{2}(t)+a x_{2}(t) x_{2}(t-\tau)\right] \leq \\
& \leq 2\left[-x_{1}^{2}(t)-(k-0.5 a) x_{2}^{2}(t)\right]+a x_{2}^{2}(t-\tau) \leq \\
& \leq-2 \min \{1, k-0.5 a\} V_{1}(t)+a V_{1}(t-\tau) .
\end{aligned}
$$

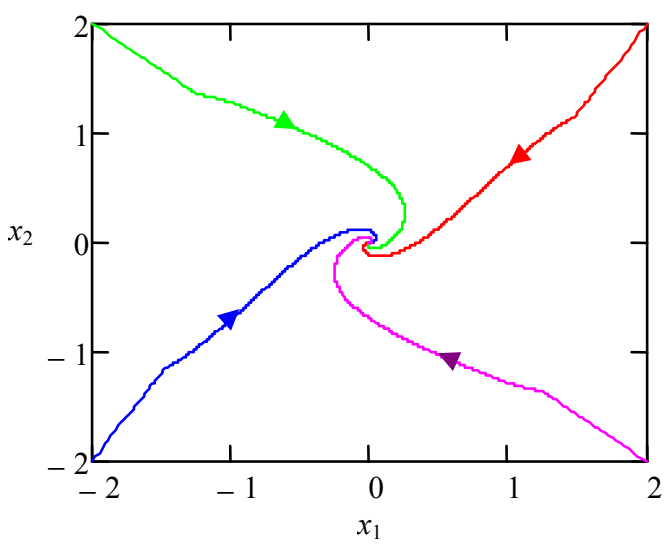

Fig. 1. The results of simulation.

Then

$$
\begin{gathered}
\max _{\theta \in[-\tau, 0]} V_{1}(\mathbf{x}(t+\theta))<a^{-1} \min \{1, k-0.5 a\} V_{1}(t) \Rightarrow \\
\dot{V}_{1} \leq-2 \min \{1, k-0.5 a\} V_{1},
\end{gathered}
$$

and if $a^{-1} \min \{1, k-0.5 a\}>1$ the system (2) at $\lambda_{1}=0$ is asymptotically stable, that according to Theorem 2 implies local asymptotic stability of the system around the origin. Obviously for $\lambda_{2}=+\infty$ the system (2) is also asymptotically stable that ensures global convergence of the trajectories to a vicinity of the origin. Note that approximate behavior of the system in this case is defined by the time-delay free dynamics (Corollary 1). The results of simulation for $k=1, a=0.5$, $\tau=0.1$ presented in Fig. 1 demonstrate that actually the system is globally asymptotically stable.

\section{CONCLUSION}

The homogeneity notion is extended to time-delay systems (nonlinear differential equations with functional arguments). The sufficient conditions for stability of homogenous systems is presented. These conditions are based on the Razumikhin 
stability arguments. It is shown on a counterexample that development of the Lyapunov-Krasovskii approach is tricky for the homogeneous case. The definition of local homogeneity is proposed, relations between stability of the locally approximating dynamics and the original system are established. Efficiency of the proposed approach is demonstrated on academic example.

\section{REFERENCES}

Andrieu V., Praly L., Astolfi A. (2008). Homogeneous Approximation, Recursive Observer Design, and Output Feedback. SIAM J. Control Optimization, 47(4), pp. 1814-1850.

Asl F.M., Ulsoy A.G. (2000). Analytical Solution of a System of Homogeneous Delay Differential Equations via the Lambert Function. Proc. American Control Conference, Chicago, Illinois, pp. 2496-2500.

Bacciotti A., Rosier L. (2001). Liapunov Functions and Stability in Control Theory. Lecture Notes in Control and Inform. Sci., 267, Springer,Berlin.

Bhat S.P., Bernstein D.S. (2005). Geometric homogeneity with applications to finite-time stability. Mathematics of Control, Signals and Systems, 17, pp. 101-127.

Bokharaie V.S., Mason O., Verwoerd M. (2010). D-Stability and Delay-Independent Stability of Homogeneous Cooperative Systems. IEEE Trans. Automatic Control, 55(12), pp. 2882-2885.

Chiasson J., Loiseau J.J. (Eds.). (2007). Applications of Time Delay Systems. Lecture Notes in Control and Information Sciences, Vol. 352, Springer.

Dublik J. (1997). Asymptotic equilibrium for homogeneous delay linear differential equations with 1-perturbation term. Nonlinear Analysis, Theory, Methods \& Applicarions, 30(6), pp. 3927-3933.

Efimov D.V., Perruquetti W. (2010). Oscillations Conditions in Homogenous Systems. Proc. NOLCOS'10, Bologna, pp. 1379-1384.

Grüne L. (2000). Homogeneous state feedback stabilization of homogeneous systems. SIAM J. Control Optimization, 38(4), pp. 1288-1314.

Gu K., Kharitonov K.L., Chen J. (2003). Stability of TimeDelay Systems (Control Engineering). Birkhäuser Boston.

Hermes H. (1991a). Nilpotent and high-order approximations of vector field systems. SIAM Review, 33(2), pp. 238-264.

Hermes H. (1991b). Homogeneous coordinates and continuous asymptotically stabilizing feedback controls. In Differential Equations: Stability and Control, Lecture Notes in Pure Appl. Math.,109, S. Elaydi, ed., Marcel Dekker,pp.249-260.

Hong Y. (2002). Finite-time stabilization and stabilizability of a class of controllable systems. Systems\&Control Lett., 46, pp. 231-236.

Kawski M. (1991). Homogeneous feedback stabilization. In Progress in systems and control theory: Vol.7. New trends in systems theory. Birkhuser.

Kolmanovsky V.B., Nosov V.R. (1986). Stability of functional differential equations. San Diego, CA: Academic.
Lin Y., Sontag E.D., Wang Y. (1996). A Smooth Converse Lyapunov Theorem for Robust Stability. SIAM J. Control Optimization, 34, pp. 124-160.

Moulay E., Perruquetti W. (2006). Finite time stability and stabilization of a class of continuous systems. J. Mathematical Analysis Applications, 323(2), pp. 1430-1443.

Richard J.P. (2003). Time-delay systems: an overview of some recent advances and open problems. Automatica, 39, pp. 1667-1694.

Rosier L. (1992). Homogeneous Lyapunov function for homogeneous continuous vector field. Systems\&Control Lett., 19, pp. 467-473.

Rothschild L.P., Stein E.M. (1976). Hypoelliptic differential operators and nilpotent groups. Acta Mathematica, 137, pp. 247-320.

Sepulchre R., Aeyels D. (1996). Stabilizability does not imply homogeneous stabilizability for controllable systems. SIAM J. Control Optimization, 34(5), pp. 1798-1813. 